\title{
MUSIELAK-ORLICZ SPACES AND PREDICTION PROBLEMS
}

\author{
K. URBANIK \\ Institute of Mathematics, Polish Academy of Sciences \\ Śniadeckich 8, P.O. Box 21, 00-956 Warszawa 10, Poland \\ E-mail: urbanik@math.uni.wroc.pl
}

Dedicated to the memory of Professor Władystaw Orlicz

\begin{abstract}
By a harmonizable sequence of random variables we mean the sequence of Fourier coefficients of a random measure $M$ :

$$
X_{n}(M)=\int_{0}^{1} e^{2 \pi n i s} M(d s) \quad(n=0, \pm 1, \ldots)
$$

The paper deals with prediction problems for sequences $\left\{X_{n}(M)\right\}$ for isotropic and atomless random measures $M$. The crucial result asserts that the space of all complex-valued $M$-integrable functions on the unit interval is a Musielak-Orlicz space. Hence it follows that the problem for $\left\{X_{n}(M)\right\}(n=0, \pm 1, \ldots)$ to be deterministic is in fact an extremal problem of Szegö's type for Musielak-Orlicz spaces in question. This leads to a characterization of deterministic sequences $\left\{X_{n}(M)\right\}(n=0, \pm 1, \ldots)$ in terms of random measures $M$.
\end{abstract}

1. Random measures and harmonizable sequences. A function $M$ defined on the $\sigma$-algebra of all Borel subsets of the unit interval $I$ whose values are complex random variables is called a random measure if

(i) for every sequence $E_{1}, E_{2}, \ldots$ of disjoint Borel sets

$$
M\left(\bigcup_{n=1}^{\infty} E_{n}\right)=\sum_{n=1}^{\infty} M\left(E_{n}\right),
$$

where the series converges with probability 1 ,

(ii) for every sequence $E_{1}, E_{2}, \ldots$ of disjoint Borel sets the random variables $M\left(E_{1}\right)$, $M\left(E_{2}\right), \ldots$ are independent.

The theory of random measures was developed by A. Prékopa in [15, 16] and [17]. For further results see [8], [22], [3] and [4].

2000 Mathematics Subject Classification: 60G25, 60G57, 46E30.

The paper is in final form and no version of it will be published elsewhere. 
A random measure $M$ is said to be atomless if $M(\{a\})=0$ with probability 1 for every one-point set $\{a\}$. Moreover a random measure $M$ is said to be isotropic if for every orthogonal transformation $U$ of the complex plane and every Borel subset $E$ of the unit interval $I$ the random variables $M(E)$ and $U M(E)$ have the same probability distribution. In particular, isotropic random measures are symmetric, i.e. for every Borel set $E$ the random variables $M(E)$ and $-M(E)$ are identically distributed. All random measures under consideration in the sequel will tacitly be assumed to be atomless and isotropic. In particular for every Borel set $E$ the random variable $M(E)$ has an infinitely divisible distribution and its characteristic function can be written in the form

$$
\varphi_{M(E)}(t)=\exp \left(\int_{0}^{\infty}\left(J_{0}(x|t|)-1\right) \frac{1+x^{2}}{x^{2}} \mu_{M}(E, d x)\right),
$$

where $J_{0}$ is the Bessel function

$$
J_{0}(x)=\frac{1}{\pi} \int_{0}^{\pi} \cos (x \sin n) d n,
$$

$\mu_{M}(E, \cdot)$ is a finite non-negative Borel measure on the positive half line $R_{+}, t \in R^{2}$ and $|t|^{2}=(t, t)$. Moreover, for every Borel subset $A$ of $R_{+}$the set-function $\mu_{M}(\cdot, A)$ is a non-negative atomless Borel measure on $I$.

In the sequel we shall identify random variables which are equal with probability 1. Given a random measure $M$, we say that a Borel set $E$ is an $M$-null set if $M(A)=0$ for all Borel subsets $A$ of $E$. Relations valid except of an $M$-null set are said to be valid $M$-almost everywhere.

By a harmonizable sequence of random variables we mean the sequence of Fourier coefficients of a random measure $M$, i.e. the sequence

$$
X_{n}(M)=\int_{0}^{1} e^{2 \pi i n s} M(d s) \quad(n=0, \pm 1, \ldots) .
$$

It is clear that the Fourier coefficients $\left\{X_{n}(M)\right\}$ determine the random measure $M$ uniquely.

A sequence $\left\{X_{n}(M)\right\}(n=0, \pm 1, \ldots)$ of random variables is called strictly stationary or, briefly, stationary, if for every system $m, n_{1}, n_{2}, \ldots, n_{k}$ of integers the multivariate distribution of the random variables

$$
X_{n_{1}+m}, X_{n_{2}+m}, \ldots, X_{n_{k}+m}
$$

does not depend upon $m$. One can prove the following result ([20], Theorem 4.1): Asequence $\left\{X_{n}(M)\right\}$ of Fourier coefficients is stationary if and only if the random measure $M$ is isotropic. In this case the probability distribution of $\left\{X_{n}(M)\right\}$ is completely determined by the set-function $\mu_{M}(\cdot, \cdot)$.

The concept of the integral with respect to a random measure was introduced in [16] (the unconditional integral) and in [22]. We shall quote the basic definition, which is an adaptation of Dunford's definition of the integral with respect to a measure whose values belong to a Banach space ([7], Chapter IV). 
If $f$ is a complex-valued Borel simple function on $I$, i.e.

$$
f=\sum_{j=1}^{n} c_{j} 1_{E_{j}}
$$

where $c_{j}$ are complex numbers and $1_{E_{j}}$ denote the indicators of Borel sets $E_{j}$, then the integral on every Borel set $E$ of $f$ with respect to the random measure $M$ is defined by the formula

$$
\int_{E} f(s) M(d s)=\sum_{j=1}^{n} c_{j} M\left(E_{j} \cap E\right) .
$$

Further, a complex-valued Borel function $g$ on $I$ is said to be $M$-integrable if there exists a sequence $\left\{g_{n}\right\}$ of simple Borel functions such that

(a) the sequence $\left\{g_{n}\right\}$ converges to $g M$-almost everywhere on $I$,

(b) for every Borel set $E$ the sequence $\left\{\int_{E} g_{n}(s) M(s)\right\}$ converges in probability.

Now, by definition, the integral $\int_{E} g(s) M(d s)$ is the limit in probability of the sequence $\left\{\int_{E} g_{n}(s) M(d s)\right\}$.

Let $L(M)$ be the set of all complex-valued $M$-integrable functions on $I$. We indentify functions which are equal $M$-almost everywhere. The space $L(M)$ is a complete linear metric space under usual addition and scalar multiplication with a non-homogeneous norm defined by the formula

$$
\|f\|_{M}=\left\|\int_{I} f(s) M(d s)\right\|,
$$

where $\|X\|$ denotes the Fréchet norm of the random variable $X$ i.e. the expectation $E(|x| /(1+|x|))$ (see [22] and [21]). It should be noted that the convergence of a sequence of functions in $L(M)$ is equivalent to the convergence in probability of the sequence of their $M$-integrals. Moreover, the set of all Borel simple functions on $I$ is dense in $L(M)$.

2. Sequences admitting a prediction. Given a stationary sequence of random variables $\left\{X_{n}\right\}$, by $\left[X_{n}\right]$ and $\left[X_{n}: n \leq k\right]$ we shall denote the linear spaces closed with respect to the convergence in probability spanned by all random variables $X_{n}$ and by random variables $X_{n}$ with $n \leq k$ respectively. To each stationary sequence $\left\{X_{n}\right\}$ there corresponds a shift transformation $T X_{n}=X_{n+1},(n=0, \pm 1, \ldots)$ which can be extended to an invertible linear transformation $T$ on $\left[X_{n}\right]$. Of course, the transformation $T$ preserves the probability distribution.

A concept of prediction for stationary sequences which need not have a finite variance was introduced in [19]. In this paper we restrict ourselves to symmetric sequences. In this case 0 is the only constant belonging to $\left[X_{n}\right]$.

We say that a stationary symmetric sequence $\left\{X_{n}\right\}$ admits a prediction if there exists a continuous linear operator $A_{0}$ from $\left[X_{n}\right]$ onto $\left[X_{n}: n \leq 0\right]$ such that

(i) $A_{0} X=X$ whenever $X \in\left[X_{n}: n \leq 0\right]$,

(ii) if for every $Y \in\left[X_{n}: n \leq 0\right]$ the random variables $X \in\left[X_{n}\right]$ and $Y$ are independent, then $A_{0} X=0$, 
(iii) for every $X \in\left[X_{n}\right]$ and $Y \in\left[X_{n}: n \leq 0\right]$ the random variables $X-A_{0} X$ and $Y$ are independent.

The random variable $A_{0} X$ can be regarded as a linear prediction of $X$ based on the full past of the sequence $\left\{X_{n}\right\}$ up to time 0 . An optimality criterion is given by (iii). In what follows the operator $A_{0}$ will be called a predictor based on the past of the sequence $\left\{X_{n}\right\}$ up to time 0 .

It should be noted that Gaussian stationary sequences with zero mean always admit a prediction. This follows from the fact that in this case the concepts of independence and orthogonality are equivalent and, moreover, the square-mean convergence and the convergence in probability are equivalent. Therefore the predictor $A_{0}$ is simply the best linear least squares predictor, i.e. the orthogonal projector from $\left[X_{n}\right]$ onto $\left[X_{n}: n \leq 0\right]$.

The predictor $A_{0}$ and the shift $T$ induced by $\left\{X_{n}\right\}$ determine the predictor $A_{k}$ based on the full past of $\left\{X_{n}\right\}$ up to time $k$ by means of the formula $A_{k}=T^{k} A_{0} T^{-k}$.

A stationary sequence $\left\{X_{n}\right\}$ admitting a prediction is called deterministic if $A_{0} X=X$ for every $X \in\left[X_{n}\right]$. Further, a stationary sequence $\left\{X_{n}\right\}$ admitting a prediction is called completely non-deterministic if for every $X \in\left[X_{n}\right]$ we have

$$
\lim _{k \rightarrow-\infty} A_{k} X=0 \text {. }
$$

It is very easy to prove that every stationary sequence admitting a prediction can be decomposed into a deterministic and a completely non-deterministic components ([19], Theorem 1). Moreover, each stationary harmonizable sequence admitting a prediction is the sum of two independent stationary harmonizable sequences admitting a prediction, one completely non-deterministic and the other deterministic ([20], Theorem 4.2). Thus the study of stationary harmonizable sequences admitting a prediction is reduced to the study of deterministic and completely non-deterministic stationary harmonizable sequences.

We note that the condition $\left[X_{n}\right]=\left[X_{n}: n \leq 0\right]$ characterizes deterministic sequences $\left\{X_{n}\right\}$. Therefore, the structure of the space $\left[X_{n}\right]$ plays a key role in our considerations. In the next section we shall quote some auxiliary concepts and a characterization of the space $L(M)$. Hence a complete description of the space $\left[X_{n}\right]$ will follow.

3. Musielak-Orlicz spaces. Given a finite measure $\nu$ defined on Borel subsets of the unit interval $I$ with $\nu(I)>0$, we take a function $\Phi$ defined on $I \times R_{+}$and satisfying the following conditions:

(3.1) $\Phi(t, 0)=0$ and $\Phi(t, x)>0$ for $x>0$ and $\nu$-almost all $t$,

(3.2) $\Phi(t, x)$ is a continuous non-decreasing function of $x$ for every $t \in I$,

(3.3) $\Phi(t, x)$ is Borel measurable as a function of $t$ for every $t \in I$,

(3.4) $\int_{I} \Phi(t, 1) \nu(d t)<\infty$,

(3.5) (the $\Delta_{2}$-condition) there exists a positive constant $c$ such that $\Phi(t, 2 x) \leq c \Phi(t, x)$ for all $x$ and $\nu$-almost all $t$.

Throughout this paper we identify functions equal $\nu$-almost everywhere. Let $f$ be a complex-valued Borel function on $I$. It is easily seen that $\Phi(t,|f(t)|)$ is also a Borel 
function on $I$. We define a modular $\rho$ by means of the formula

$$
\rho(f)=\int_{I} \Phi(t,|f(t)|) \nu(d t) .
$$

Let $L_{\Phi}(\nu)$ be the set of all complex-valued Borel functions $f$ on $I$ for which $\rho(f)$ is finite. The set $L_{\Phi}(\nu)$ is a linear space over the complex field under usual addition and scalar multiplication. Moreover, it becomes a complete linear metric space under the non-homogeneous norm

$$
\|f\|=\inf \left\{a: a>0, \rho\left(a^{-1} f\right) \leq a\right\} .
$$

The space $L_{\Phi}(\nu)$ with this norm was introduced and investigated by J. Musielak and W. Orlicz in [14] and will be called a Musielak-Orlicz space. From (3.4) it follows that all bounded Borel functions on $I$ belong to $L_{\Phi}(\nu)$. Moreover, the set of all Borel simple functions is dense in $L_{\Phi}(\nu)$.

In this paper two linear metric spaces $\left(Y,\|\|_{1}\right)$ and $\left(Y,\|\|_{2}\right)$ will be treated as identical if the convergences in both norms \|\|$_{1}$ and \|\|$_{2}$ are equivalent. In particular, if

$$
a \Phi(t, x) \leq \Psi(t, x) \leq b \Psi(t, x)
$$

for some positive numbers $a$ and $b, \nu$-almost all $t$ and sufficiently large $x$, then $L_{\Phi}(\nu)=$ $L_{\Psi}(\nu)$. Moreover, if $\beta(t)>0$ for $t \in I, \int_{I} \beta(s) \nu(d s)<\infty, \Phi(t, x)=\Psi(t, x) / \beta(t)$ and $\lambda(E)=\int_{E} \beta(s) \nu(d s)$, then $L_{\Psi}(\nu)=L_{\Phi}(\nu)$. Therefore, without loss of generality, we may always assume that

$$
\Phi(t, 1)=1 \quad \text { for } t \in I .
$$

Let $K$ be the class of all pairs $(\Phi, \nu)$ satisfying conditions (3.1)-(3.5) such that the measure $\nu$ is atomless and for $\nu$-almost all $t$ the function $\Phi(t, \sqrt{x})$ is concave on $R_{+}$.

Given a random measure $M$ we denote by $\mu_{M}(\cdot, \cdot)$ the corresponding set-function appearing in formula (1.1). Put

$$
\nu_{M}(E)=\mu_{M}\left(E, R_{+}\right)
$$

for every Borel subset $E$ of $I$. It is obvious that all measures $\mu_{M}(\cdot, A)$ are absolutely continuous with respect to the measure $\nu_{M}$. Consequently, by the Radon-Nikodym Theorem,

$$
\mu_{M}(E,[0, x))=\int_{E} g_{M}(s, x) \nu_{M}(d s),
$$

where $0 \leq g_{M}(s, x) \leq 1$ and the function $g_{M}(\cdot, x)$ is Borel measurable on $I$. Moreover, we may assume, without loss of generality, that the function $g_{M}(s, \cdot)$ is monotone nondecreasing and continuous to the left on $R_{+}$. Put

$$
\Phi_{M}(t, x)=\int_{1 / x}^{\infty} \frac{g_{M}(t, u)}{u^{3}} d u \quad\left(t \in I, x \in R_{+}\right) .
$$

By a simple calculation we have

$$
\Phi_{M}(t, \sqrt{x})=\frac{1}{2} \int_{0}^{x} g_{M}\left(t, \frac{1}{\sqrt{u}}\right) d u
$$

and, consequently, $\left(\Phi_{M}, \nu_{M}\right) \in K$. 
We shall lean heavily on the following representation of the space $L(M)$ of $M$ integrable functions, which provides a tool for investigating random harmonizable sequences ([20], Theorem 3.1).

TheOREM 3.1. For every random measure $M$ we have the relations $\left(\Phi_{M}, \nu_{M}\right) \in K$ and $L(M)=L_{\Phi_{M}\left(\nu_{M}\right)}$.

The converse implication is also true.

TheOREM 3.2. For every pair $(\Phi, \nu) \in K$ there exists a random measure $M$ such that $L(M)=L_{\Phi}(\nu)$.

Proof. Let $(\Phi, \nu) \in K$. Without loss of generality we may assume that condition (3.6) holds. Put

$$
\Phi(t, \sqrt{x})=\int_{0}^{x} q(t, u) d u \quad(t \in I),
$$

where $q(t,$.$) is a non-negative monotone non-increasing function. Setting r(t, u)=q(t, u)$ for $u>1$ and $r(t, u)=1$ for $0 \leq u \leq 1$ we get a non-negative monotone non-increasing function $r(t,$.$) . Moreover, the function$

$$
\Psi(t, x)=\int_{0}^{x^{2}} r(t, u) d u
$$

fulfils the condition $\Phi(t, x)=\Psi(t, x)$ for $x \geq 1$ and $(\Psi, \nu) \in K$. Consequently,

$$
L_{\Psi}(\nu)=L_{\Phi}(\nu) .
$$

Now we shall prove that there exists a random measure $M$ fulfilling the condition

$$
\mu_{M}(E,[0, x))=\int_{E} r\left(s, x^{-2}\right) \nu(d s) .
$$

In fact, for the set-function (3.9) there exists a separable stochastic process with independent increments such that the characteristic function of the increment $X(b)-X(a)$ is given by the expression

$$
\exp \left(\int_{0}^{\infty}\left(J_{0}(x|t|-1) \frac{1+x^{2}}{x^{2}} \mu_{M}([a, b), d x)\right),\right.
$$

(see [6], p. 61 and 418). Setting $M\left(\bigcup_{j=1}^{n}\left[a_{j}, b_{j}\right)\right)=\sum_{j=1}^{n}\left(X\left(b_{j}\right)-X\left(a_{j}\right)\right)$ for disjoint intervals $\left[a_{j}, b_{j}\right)(j=1,2, \ldots, n)$ we get a random set function which, by Prékopa's Theorems ([15], p.227, 243) can be extended to a random measure $M$ defined on Borel subset of $I$. Further, from (3.9) we get $\nu_{M}=\nu$ and $g_{M}(t, x)=r\left(t, x^{-2}\right)$ which, by (3.7) yields the equality

$$
\Phi_{M}(t, x)=\frac{1}{2} \Psi(t, x)
$$

Consequently, $L_{\Phi_{M}}\left(\nu_{M}\right)=L_{\Psi}(\nu)$ and, by (3.8) and Theorem 3.1, $L(M)=L_{\Phi}(\nu)$. The theorem is thus proved.

In attempting to visualize these representation theorems we shall give some examples. 
Example 3.1. We say that $M$ is a random Poisson measure if there exists a finite Borel measure $\beta(\cdot, \cdot)$ on $I \times R_{+}$such that

$$
\mu_{M}(E, d x)=\frac{x^{2}}{1+x^{2}} \beta(E, d x) .
$$

Integrating by parts it is easy to verify that

$$
\begin{aligned}
\int_{I} \Phi_{M}(t, x) \nu_{M}(d t) & =\int_{1 / 2}^{\infty} \int_{I} g_{M}(t, u) \nu_{M}(d t) \frac{d u}{u^{3}} \\
& =\frac{1}{2} \int_{0}^{\infty} \frac{\min \left(x^{2} u^{2}, 1\right)}{1+u^{2}} \beta(I, d u) \leq \beta\left(I, R_{+}\right)
\end{aligned}
$$

for every $x \in R_{+}$. Consequently, $\Phi_{M}(t, \cdot)$ are bounded for $\nu$-almost every $t \in I$.

ExAmple 3.2. Given $p \geq 0$ and an atomless measure $\nu$ on $I$ we put

$$
\mu_{M}(E, d x)=2 \lambda(E) p^{-p} e^{p} x\left(1+e^{p} x^{-2}\right)^{-2} \log ^{p-1}\left(e^{p}+x^{-2}\right) \log \left(1+e^{p} x^{-2}\right) d x
$$

Then $\nu_{M}=\lambda$ and

$$
\Phi_{M}(t, x)=e^{p} p^{-p} 2^{-1}(1+p)^{-1}\left(\log ^{1+p}\left(e^{p}+x^{2}\right)-p^{1+p}\right) .
$$

EXAMPLE 3.3. Let $\lambda$ be an atomles measure on $I$ and

$$
\mu_{M}(E, d x)=2 \lambda(E) x\left(1+e x^{2}\right)^{-2}\left(\log \log \left(e+x^{-2}\right)+1-\log ^{-1}\left(e+x^{-2}\right)\right) .
$$

Then

$$
\Phi_{M}(r, x)=\frac{e}{2} \log \left(e+x^{2}\right) \log \log \left(e+x^{2}\right) .
$$

ExAmPLE 3.4. Given $0<p<2$ and an atomless measure $\nu$ we put

$$
\mu_{M}(E, d x)=\beta \nu(E) \frac{x^{1-p}}{1+x^{2}} d x,
$$

where $\beta=\frac{2}{\pi} \sin \frac{p \pi}{2}$. Here we have $\nu_{M}=\nu$ and the measure $\mu_{M}$ corresponds to a $p$-stable random measure $M$ with the characteristic function $\varphi_{M(E)}(t)=\exp \left(-\nu(E)|t|^{p}\right)$. It is easy to check that

$$
a x^{p} \leq \Phi_{M}(t, x) \leq b x^{p} \quad\left(t \in I, x \in R_{+}\right)
$$

for some positive constants $a$ and $b$. Thus $L(M)=L^{p}(\nu)$.

Consider a stationary harmonizable sequence $\left\{X_{n}(M)\right\}(n=0, \pm 1, \ldots)$ corresponding to a random measure $M$. It is easy to verify that the mapping

$$
X_{n}(M) \rightarrow e^{2 \pi n i s} \quad(n=0, \pm 1, \ldots, s \in I)
$$

can be extended in a natural way to an isomorphism between $\left[X_{n}(M)\right]$ and $L(M)$. Moreover,

$$
\left[X_{n}(M)\right]=\left\{\int_{I} f(s) M(d s): f \in L(M)\right\}
$$

and by Theorem 3.1, formula (3.10) defines a natural isomorphism from $\left[X_{n}(M)\right]$ onto the Musielak-Orlicz space $L_{\Phi_{M}}\left(\nu_{M}\right)$. It is evident that the sequence $\left\{X_{n}(M)\right\}$ is deterministic if and only if

$$
X_{0}(M) \in\left[X_{n}(M): n \leq-1\right]
$$


Denoting by \|\| the norm in $L_{\Phi_{M}}\left(\nu_{M}\right)$, we infer that $\left\{X_{n}(M)\right\}$ is deterministic if and only if

$$
\inf \left\|1+\sum_{k=1}^{n} a_{k} e^{-2 \pi k i s}\right\|=0
$$

where the infimum is taken over all complex numbers $a_{1}, a_{2}, \ldots, a_{n}$ and $n=1,2, \ldots$ Since $\|f\|=\|\bar{f}\|$, we observe that (3.11) is equivalent to the relation

$$
\inf \left\|1+\sum_{k=1}^{n} a_{k} e^{2 \pi k i s}\right\|=0 .
$$

A solution of this extremal problem of Szegö's type can be regarded as a generalisation of the famous Kolmogorov-Krein criterion for $L^{p}$-spaces $([9,10])$. This question will be discussed in the next section.

4. An extremal problem for Musielak-Orlicz spaces. Given a Borel measure $\nu$ on $I$ by $\nu_{c}$ we shall denote the absolutely continuous component of $\nu$ with respect to the Lebesgue measure and by $d \nu_{c} / d t$ a Borel measurable version of its Radon-Nikodym density function. For any pair $(\Phi, \nu)$ satisfying conditions (3.1)-(3.6) we introduce auxiliary functions $\lambda_{\Phi, \nu}$ and $\Omega_{\Phi, \nu, n}(n=1,2, \ldots)$ by means of the formulas

$$
\begin{aligned}
& \Lambda_{\Phi, \nu}(t, x)=\sup \left\{\frac{\log y}{\Phi(t, y)}\left(\frac{d \nu_{c}}{d t}\right)^{-1}: y \geq x\right\}, \\
& \Omega_{\Phi, \nu, n}(t)=\inf \left\{x: \Lambda_{\Phi, \nu}(t, x) \leq n, x \geq 1\right\},
\end{aligned}
$$

where the infimum of an empty set is defined as $\infty$. It is clear that all these functions are Borel measurable and $1 \leq Q_{\Phi, \nu, n}(t) \leq \infty(n=1,2, \ldots)$.

The following generalization of the Kolmogorov-Krein criterion was proved in [20] (Theorem 1.1).

TheOREM 4.1. Let $L_{\Phi}(\nu)$ be a Musielak-Orlicz space with the norm \|\| . The equation

$$
\inf \left\|1+\sum_{k=1}^{n} a_{k} e^{2 \pi k i t}\right\|=0,
$$

where the infimum is taken over all complex numbers $a_{1}, a_{2}, \ldots, a_{n}$ and $n=1,2, \ldots$, holds if and only if no function

$$
\log \Omega_{\Phi, \nu, n} \quad(n=1,2, \ldots)
$$

is Lebesgue integrable over I.

Now we shall quote some particular cases of this theorem. Given a number $b>1$, we say that a function $\Phi$ satisfies the $\Lambda_{b}$-condition if there exists a constant $e_{b}>1$ and a positive number $x_{0}$ such that

$$
\Phi(t, x) e_{b} \leq \Phi(t, b x)
$$

for all $t \in I$ and $x \geq x_{0}$ (see [13]). 
TheOREM 4.2. Let $L_{\Phi}(\nu)$ be a Musielak-Orlicz space satisfying the $\Lambda_{b}$-condition for some constant $b>1$. Then equation (4.1) holds if and only if $\log \frac{d \nu_{c}}{d t}$ is not Lebesgue integrable over $I$.

Proof. From the $\Lambda_{b}$-condition it follows that there are positive constants $c_{1}$ and $p$ such that

$$
c_{1} x^{p} \leq \Phi(t, x)
$$

for sufficiently large $x$ and all $t \in I$ (see [13], 124). Further, from the $\Delta_{2}$-condition (3.5) it follows that there are positive constants $c_{2}$ and $q$ such that

$$
\Phi(t, x) \leq c_{2} x^{q}
$$

for sufficiently large $x$ and $\nu$-almost all $t$. Consequently, we can find a positive number $x_{0}>1$ such that

$$
c_{1} x^{p} \leq \frac{\Phi(t, x)}{\log x} \leq c_{2} x^{q}
$$

for all $x \geq x_{0}$ and $\nu$-almost all $t$. Hence in particular it follows that

$$
\lim _{x \rightarrow \infty} \Lambda_{\Phi, \nu}(t, x)=0
$$

$\nu$-almost everywhere. Consequently, the functions $\Omega_{\Phi, \nu, n}(n=1,2, \ldots)$ are finite $\nu$-almost everywhere.

Suppose first that the Lebesgue measure is not absolutely continuous with respect to the measure $\nu$. Then $\frac{d \nu_{c}}{d t}$ vanishes on a set of positive Lebesgue measure. Consequently, all functions $\Omega_{\Phi, \nu, n}(n=1,2, \ldots)$ and the function $\log \frac{d \nu_{c}}{d t}$ are infinite on a set of positive Lebesgue measure, which, by Theorem 4.1, implies our assertion.

Now suppose that the Lebesgue measure is absolutely continuous with respect to the measure $\nu$. Then the functions $\Omega_{\Phi, \nu, n}(n=1,2, \ldots)$ are finite almost everywhere in the sense of the Lebesgue measure. Moreover, inequality (4.2) holds also for all $x \geq x_{0}$ and for almost all $t$ in the sense of Lebesgue measure. Put

$$
F_{n}=\left\{t: x_{0}<\Omega_{\Phi, \nu, n}(t)<\infty\right\} \quad(n=1,2, \ldots) .
$$

It is very easy to verify that both functions $\log \frac{d \nu_{c}}{d t}$ and $\log \Omega_{\Phi, \nu, n}(t)$ are Lebesgue integrable over $I \subset F_{n}$. Moreover, for all $t \in F_{n}$ we have the formula

$$
\log \Omega_{\Phi, \nu, n}(t)\left(\frac{d \nu_{c}}{d t}\right)^{-1}=n \Phi\left(t, \Omega_{\Phi, \nu, n}(t)\right) .
$$

Hence and from (4.2) we get the inequality

$$
n c_{1} \Omega_{\Phi, \nu, n}^{p}(t) \leq\left(\frac{d \nu_{c}}{d t}\right)^{-1} \leq n c_{2} \Omega_{\Phi, \nu, n}^{q}(t)
$$

for almost all $t$ from $F_{n}$ in the sense of the Lebesgue measure. Consequently, the function $\log \frac{d \nu_{c}}{d t}$ and all the functions $\log \Omega_{\Phi, \nu, n}$ simultaneously are not Lebesgue integrable over $I$ which, by Theorem 4.1, completes the proof.

TheOREm 4.3. Let $L_{\Phi}(\nu)$ be a Musielak-Orlicz space satisfying the condition

$$
\lim _{x \rightarrow \infty} \frac{\Phi(t, x)}{\log x}=0
$$

on a set of positive Lebesgue measure. Then equation (4.1) is fulfilled. 
Proof. It is very easy to verify that $\Omega_{\Phi, \nu, n}(t)=\infty$ for all $t$ from $I$ satisfying (4.3) and the inequality $\frac{d \nu_{c}}{d t}<\infty$. Since the density function $\frac{d \nu_{c}}{d t}$ is finite almost everywhere with respect to the Lebesgue measure, we conclude that no function $\log \Omega_{\Phi, \nu, n}$ is Lebesgue integrable over $I$ which, by Theorem 4.1, gives formula (4.1).

In the same way one can prove the following theorems.

THEOREM 4.4. Let $L_{\Phi}(\nu)$ be a Musielak-Orlicz space satisfying for some positive numbers $a$ and $b$ the condition

$$
a \leq \frac{\Phi(t, x)}{\log x} \leq b
$$

for $x \geq x_{0}$ and almost all $t$ in the sense of the Lebesgue measure. Then equation (4.1) holds if and only if ess inf $\frac{d \nu_{c}}{d t}=0$.

TheOREM 4.5. Let $L_{\Phi}(\nu)$ be a Musielak-Orlicz space. If there are positive numbers $a, b, p$ and $x_{0}$ such that

$$
a \leq \frac{\Phi(t, x)}{\log ^{1+p} x} \leq b
$$

for $x \geq x_{0}$ and almost all $t$ in the sense of the Lebesgue measure, then equation (4.1) holds if and only if

$$
\int_{I}\left(\frac{d \nu_{c}}{d t}\right)^{-1 / p} d t=\infty .
$$

THEOREM 4.6. Let $L_{\Phi}(\nu)$ be a Musielak-Orlicz space. If there are positive numbers a,b and $x_{0}$ such that

$$
a \leq \frac{\Phi(t, x)}{\log x \log \log x} \leq b,
$$

for $x \geq x_{0}$ and almost all $t$ in the sense of the Lebesgue measure, then equation (4.1) holds if and only if

$$
\int_{I} \exp \left\{n^{-1}\left(\frac{d \nu_{c}}{d t}\right)^{-1}\right\} d t=\infty
$$

for all positive integers $n$.

5. Deterministic harmonizable sequences. We proceed now to a description of stationary harmonizable sequences $\left\{X_{n}(M)\right\}$ in terms of probabilistic characteristics of the random measure $M$. We recall that to every random measure $M$ there corresponds a Borel measure $\nu_{M}$ on $I$ and a function $\Phi_{M}$ on $I \times R_{+}$and the pair $\left(\Phi_{M}, \nu_{M}\right)$ determines the sequence of functions $\Omega_{\Phi, \nu, n}(n=1,2, \ldots)$ on $I$. We already know that the sequence $\left\{X_{n}(M)\right\}$ is deterministic if and only if equation (4.1) holds in $L_{\Phi_{M}}\left(\nu_{M}\right)$. Consequently, Theorem 4.1 yields the following characterization of deterministic sequences.

THEOREM 5.1. A stationary harmonizable sequence $\left\{X_{n}(M)\right\}$ is deterministic if and only if no function $\log \Omega_{\Phi_{M}, \nu_{M}, n}(n=1,2, \ldots)$ is Lebesgue integrable over I.

We illustrate this theorem by some examples. 
ExAmple 5.1. Comparing Example 3.1 and Theorem 4.3 we conclude that stationary harmonizable sequences $\left\{X_{n}(M)\right\}$ induced by random Poisson measures $M$ are always deterministic.

ExAmple 5.2. Taking into account Example 3.4 and Theorem 4.2 we infer that a stationary harmonizable sequence $\left\{X_{n}(M)\right\}$ corresponding to a $p$-stable random measure $M$ with $0<p<2$ and $\nu_{M}=\nu$ is deterministic if and only if $\log \frac{d \nu_{c}}{d t}$ is not Lebesgue integrable over $I$.

EXAmple 5.3. Consider a stationary harmonizable sequence $\left\{X_{n}(M)\right\}$ corresponding to the measure $M$ appearing in Example 3.2 with $p=0$ and $\nu_{M}=\nu$. By Theorem 4.4 this sequence is deterministic if and only if ess inf $\frac{d \nu_{c}}{d t}=0$.

EXAMPLE 5.4. Taking a stationary harmonizable sequence $\left\{X_{n}(M)\right\}$ corresponding to the measure $M$ appearing in Example 3.2 with $p>0$ and $\nu_{M}=\nu$ we infer, by Theorem 4.5 , that $\left\{X_{n}(M)\right\}$ is deterministic if and only if

$$
\int_{I}\left(\frac{d \nu_{c}}{d t}\right)^{-1 / p} d t=\infty
$$

ExAmple 5.5. Let $M$ be the random measure described by Example 3.3 with $\nu_{M}=\nu$. Applying Theorem 4.6 we conclude that the sequence $\left\{X_{n}(M)\right\}$ is deterministic if and only if

$$
\int_{I} \exp \left\{n^{-1}\left(\frac{d \nu_{c}}{d t}\right)^{-1}\right\} d t=\infty
$$

for all positive integers $n$.

6. Completely non-deterministic harmonizable sequences. First we shall quote a continuous analogue of the Bernstein-Darmois Theorem ([1], [5]), which is a main tool in the study of completely non-deterministic sequences. For homogeneous random measures this problem was discussed in [11], [18] and [21]. The following theorem was proved in [20] (Theorem 2.1).

THEOREM 6.1. Let $f$ and $g$ be $M$-integrable functions with respect to a random measure. If the random variables $\int_{I} f(s) M(d s)$ and $\int_{I} g(s) M(d s)$ are independent, then for every Borel subset $E$ of the set $\{s: f(s) g(s) \neq 0\}$ the random variable $M(E)$ is Gaussian.

Here the degenerate case $M(E)=0$ is also treated as the Gaussian one. Further, a random measure $M$ is said to be Gaussian if for every Borel subset $E$ of $I$ the random variable $M(E)$ is Gaussian. If in addition $M(I)$ does not vanish with probability 1 we have $L(M)=L^{2}\left(\nu_{M}\right)$. The classical characterization of completely non-deterministic wide sense stationary sequences ([6], Chapter XII, 4) implies the following statement.

TheOREM 6.2. Let $M$ be a Gaussian random measure. The sequence $\left\{X_{n}(M)\right\}$ is completely non-deterministic if and only if either $M \equiv 0$ with probability 1 or the measure $\nu_{M}$ is absolutely continuous with respect to the Lebesgue measure and $\log \frac{d \nu_{M}}{d t}$ is Lebesgue integrable over $I$.

A complete description of stationary harmonizable completely non-deterministic sequences is given by the following theorem. 
TheOREM 6.3. A stationary harmonizable sequence $\left\{X_{n}(M)\right\}$ is completely non-deterministic if and only if either $M \equiv 0$ with probability 1 or the measure $M$ is Gaussian, $\nu_{M}$ is absolutely continuous with respect to the Lebesgue measure and $\log \frac{d \nu_{M}}{d t}$ is Lebesgue integrable over $I$.

Proof. By Theorem 6.2 it suffices to prove that the measure $M$ is Gaussian provided $\left\{X_{n}(M)\right\}$ is completely non-deterministic.

Let $A_{k}$ be the predictor based on the full past of $X_{n}(M)$ up to time $k$. Since

we have the formula

$$
\left[X_{n}(M)\right]=\left\{\int_{I} f(s) M(d s): f \in L(M)\right\},
$$

$$
A_{k} X_{0}(M)=\int_{I} f_{k}(s) M(d s)
$$

where $f_{k} \in L(M)$. Setting

$$
E_{k}=\left\{s: f_{k}(s) \neq 1\right\}
$$

we get the formula

$$
A_{k} X_{0}(M)=\int_{E_{k}} f_{k}(s) M(d s)+M\left(I \backslash E_{k}\right) .
$$

Of course, the random variables $M\left(I \backslash E_{k}\right)$ and $\int_{E_{k}} f_{k}(s) M(d s)$ are independent and symmetrically distributed. Consequently, the relation

$$
\lim _{k \rightarrow-\infty} A_{k} X_{0}(M)=0
$$

implies the relation

$$
\lim _{k \rightarrow-\infty} M\left(I \backslash E_{k}\right)=0 .
$$

By the definition of predictors the random variables $X_{0}(M)-A_{k} X_{0}(M)$ and $X_{k}(M)$ are independent. In other words, the integrals

$$
\int_{I}\left(1-f_{k}(s)\right) M(d s) \text { and } \int_{I} e^{2 \pi k i s} M(d s)
$$

are independent. Since both integrands are different from 0 on $E_{k}$, we infer, by Theorem 6.1, that the random $M\left(E_{k}\right)$ is Gaussian. Hence and from (6.1) it follows that $M(I)$, being the limit in probability of Gaussian random variables $M\left(E_{k}\right)$, is Gaussian too. By Cramér's Theorem ([12], p. 271), $M$ is a Gaussian random measure, which completes the proof.

\section{References}

[1] S. Bernstein, Sur la propriété caractéristique de la loi de Gauss, Trans. Leningrad Polytech. Inst. 3 (1941), 21-22.

[2] J. Bretagnolle, D. Dacunha-Castelle and J. L. Krivine, Lois stables et espaces $L^{p}$, Annales de l'Institut Henri Poincaré 2 (1966), 231-259.

[3] J. Bretagnolle and D. Dacunha-Castelle, Mesures aléatoires et espaces d'Orlicz, Comptes Rendus 264 (1967), 877-880. 
[4] J. Bretagnolle and D. Dacunha-Castelle, Formes linéaires aléatoires et plongements d'espaces de Banach dans des espaces $L^{1}$, Comptes Rendus 265 (1967), 474-477.

[5] G. Darmois, Sur une propriété caractéristique de la loi de probabilité de Laplace, Comptes Rendus 232 (1951), 1999-2000.

[6] J. L. Doob, Stochastic Processes, New York, 1953.

[7] N. Dunford and J. T. Schwartz, Linear Operators, Part I: General Theory, 1958.

[8] J. F. C. Kingman, Completely random measures, Pacific Journal of Math. 21 (1967), 59-78.

[9] A. N. Kolmogorov, Stationary sequences in Hilbert spaces, Bull. MGU 6 (1941), 1-40.

[10] M. G. Krein, On a generalization of some investigations of G.Szegö, V. I. Smirnov and A. N. Kolmogorov, Doklady Acad. Sci. U.R.S.S. 46 (1945), 91-94.

[11] R. G. Laha and E. Lukacs, On characterization of the Wiener process, in: Transactions of the Second Prague Conference on Information Theory, Statistical Decision Functions and Random Processes, 1960, 307-312.

[12] M. Loeve, Probability Theory, New York, 1950.

[13] W. Matuszewska, Przestrzenie funkcji $\varphi$-całkowalnych I, własności ogólne $\varphi$-funkcji $i$ klas funkcji $\varphi$-calkowalnych, Prace Matematyczne 6 (1961), 121-139.

[14] J. Musielak and W. Orlicz, On modular spaces, Studia Mathematica 18 (1959), 49-65.

[15] A. Prekopa, On stochastic set functions I, Acta Math. Acad. Scient. Hung. 7 (1956), 215-263.

[16] A. Prekopa, On stochastic set functions II, Acta Math. Acad. Scient. Hung. 8 (1957), 337-374.

[17] A. Prekopa, On stochastic set functions III, Acta Math. Acad. Scient. Hung. 8 (1957), $375-400$.

[18] V. P. Skitovich, On characterising Brownian motion, Teor. Verojatnost. i Primenen. 1 (1956), 361-364.

[19] K. Urbanik, Prediction of strictly stationary sequences, Coll. Math. 12 (1964), 115-129.

[20] K. Urbanik, Random measures and harmonizable sequences, Studia Mathematica 31 (1968), 61-88.

[21] K. Urbanik, Some prediction problems for strictly stationary processes, in: Proceedings of the Fifth Berkeley Symposium on Mathematical Statistics and Probability, 1967, Vol. II, Part I, 235-258.

[22] K. Urbanik and W. A. Woyczyński, A random integral and Orlicz spaces, Bull. Acad. Pol. Sci. Série Sci. Math. Astr. Phys. 15 (1967), 161-169. 